\title{
Impact of intrinsic point defect concentration on thermal transport in titanium dioxide
}

\author{
Brian F. Donovan ${ }^{\mathrm{a}, \mathrm{b}, *}$, Daniel M. Long ${ }^{\mathrm{c}}$, Ali Moballegh ${ }^{\mathrm{c}}$, Nicole Creange $^{\mathrm{c}}$, Elizabeth \\ C. Dickey ${ }^{\mathrm{c}}$, Patrick E. Hopkins ${ }^{\mathrm{b}}$ \\ ${ }^{a}$ Department of Physics, United States Naval Academy, Annapolis, Maryland 21401, USA \\ ${ }^{b}$ Department of Mechanical and Aerospace Engineering, University of Virginia, Charlottesville, Virginia \\ 22904, USA \\ ${ }^{c}$ Department of Materials Science and Engineering, North Carolina State University, Raleigh, North \\ Carolina 27695, USA
}

\begin{abstract}
The thermal conductivity of functional oxide materials can be significantly impacted by variations in point defect concentration, especially at high concentrations where defect interactions can result in extended defects and secondary phase formation. In this work, we systematically study the impact of high point defect concentrations on thermal transport in rutile $\mathrm{TiO}_{2}$. Using atmospherically controlled annealing, we vary equilibrium point defect concentrations and measure the resulting thermal conductivity using time domain thermoreflectance. We verify our results with analytical modeling and find that it is not until very high defect concentrations $(>0.5 \mathrm{~mol} . \%)$ that the phonon thermal conductivity is impacted. We vary the partial pressure of oxygen to low enough levels that sub-stoichiometric Magnéli phases form and find that these highly defective phases severely reduce the thermal conductivity and anisotropy from intrinsic levels.
\end{abstract}

Keywords: Thermal conductivity, $\mathrm{TiO}_{2}$, Point defects, Wadsley defects, Magnéli phases

* Corresponding author

Email addresses: bdonovan@usna . edu (Brian F. Donovan), ecdickey@ncsu . edu (Elizabeth C. Dickey), phopkins@virginia.edu (Patrick E. Hopkins)

Preprint submitted to Acta Materialia

January 10, 2017

(C) 2017. This manuscript version is made available under the Elsevier user license http://www.elsevier.com/open-access/userlicense/1.0/ 


\section{Introduction}

Dielectric degradation and breakdown are major reliability issues in microelectronics devices [1,2]. Since increases in temperature can significantly accelerate dielectric breakdown, understanding the evolution of the intrinsic thermal properties of dielectric materials under prolonged electric fields is of critical importance to addressing this failure mechanism [3,4]. The local point defect concentration is a material property that is subject to evolution during exposure to electric fields $[5,6,7,8,9]$ and can have a significant impact on thermal transport.

With increased concentration of point defects, thermal carrier propagation will be disrupted and local hotspots may lead to increased probability of device failure $[10,11,12]$. In the region of the electrodes of a dielectric material under prolonged electric fields, point defect concentrations can increase well beyond initial, homogeneous concentrations and lead to highly defective layers that can even move into entirely new phases from the severe non-stoichiometry $[13,8]$.

The impact of this defect buildup on thermal transport can be assessed in a systematic way by analyzing systems with similar levels of homogenous defect concentrations. The literature clearly outlines the general trend of reduced phonon transport with high point defect concentrations in a crystal $[14,15]$. The analytical form of this increase in phonon scattering with point defects has been previously determined not only for elemental crystals, but for oxides as well $[16,17]$. In oxides, not only are substitutional (alloying or doping) defects important, but non-stoichiometric point defects due to equilibration with the external oxygen activity $\left(p \mathrm{O}_{2}\right)$ must be considered as well.

Previous studies have investigated the impact of these intrinsic defects, such as oxygen vacancies, on bulk thermal transport at dilute concentrations $[18,19]$. Recently, Brooks et al. [20] have conducted a similar study using thin films, relying on molecular beam epitaxy to control the stoichiometry of constituent atomic species incident to the sample during growth, fabricating severely non-stoichiometric samples with secondary phases. However, there has yet to be a systematic experimental study that takes into account the thermal impact of the environmentally driven interaction of various 
intrinsic point defects in oxide single crystals, pushing defect concentrations beyond known trends in homogenous concentrations and into the regime of defect ordering and secondary phase formation. Studies addressing the change in thermal conductivity of severely non-stoichiometric bulk oxides with secondary phases have concentrated

35 on polycrystalline materials [21]. The strong crystallographic dependance of these non-stoichiometric secondary phases compels the present study of high defect concentrations in single crystalline materials.

Control over defect profiles in bulk, single crystal oxides can be accomplished by dictating the partial pressure of oxygen during annealing to modify oxygen activity and alter relative equilibrium concentrations of point defects [22]. This technique can be used to produce single crystals with point defect concentrations that span the range of the variable defect concentrations found within a dielectric during degradation and breakdown. The information gleaned from this study can then be used to model the evolution of thermal transport under prolonged electric fields and determine the threshold at which the local thermal conductivity will have an impact on thermal runaway and dielectric breakdown.

This work aims to experimentally elucidate the impact of intrinsic defect concentration on thermal transport by manipulating bulk point defect concentrations in rutile $\mathrm{TiO}_{2}$, an important dielectric material. We use analytical models to further understand the trends in thermal transport and identify the threshold of the impact of defectivity on the thermal conductivity as well as the impact of new phase formation at very high defect concentrations.

\section{Experimental Procedure}

The rutile single crystals used in this study ([100] and [001] orientation) are purchased from the MTI corporation $(10 \mathrm{~mm} \times 10 \mathrm{~mm} \times 0.5 \mathrm{~mm})$. We anneal each crystal at $1100^{\circ} \mathrm{C}$ for $36 \mathrm{~h}$ at varying partial pressures of oxygen $\left(p \mathrm{O}_{2}\right)$ in order to control the defect concentrations. In order to precisely control the $p \mathrm{O}_{2}$, we vary the flow rate of an $\mathrm{Ar} / \mathrm{H}_{2}$ forming gas mixture in the annealing furnace. We quench the crystals in the forming gas environment to room temperature using a sealed interlock. We vary the 


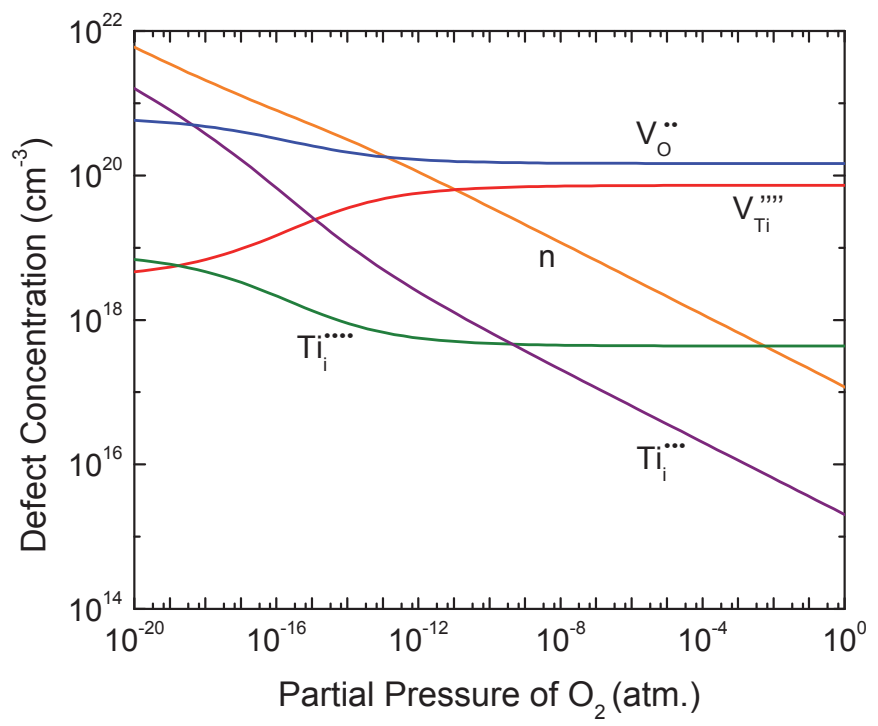

Figure 1: Concentration of primary point defects in $\mathrm{TiO}_{2}$ at room temperature dictated by partial pressure of oxygen in $1100{ }^{\circ} \mathrm{C}$ anneal. The defects, shown in Kroger-Vink notation, are oxygen vacancies, $\mathrm{V}_{\mathrm{O}}^{\bullet \bullet}$, conducting electrons, $\mathrm{n}$, titanium interstitials with $3+$ valence, $\mathrm{Ti}_{\mathrm{i}}^{\bullet \bullet \bullet}$, titanium interstitials with $4+$ valence, $\mathrm{Ti}_{\mathrm{i}}^{\bullet \bullet \bullet \bullet}$, and titanium vacancies, $\mathrm{V}_{T i}^{\prime \prime \prime \prime}$.

${ }_{60} \mathrm{pO}_{2}$ during the anneal to range from $1.7 \times 10^{-12}$ to $8.4 \times 10^{-18} \mathrm{~atm}$.

Using the well-established defect reactions and mass action relations, shown in Table 1 , we calculate the concentration of various species of point defects during high temperature equilibration $[23,24,25,26]$. The major populations of point defects present in the investigated regime are oxygen vacancies, titanium interstitials, and titanium vacancies. During the quench process, to be consistent with defect models, we assume the population density of ionic defects to be fixed and only the valence of titanium interstitials and the populations of intrinsic electronic carriers are allowed to change. The defect relations controlling the process, derived from high temperature conditions and the previous assumptions, are detailed in Table 1. The calculated concentrations of these defects, along with the concentrations of the electrons and holes can be seen in Fig. 1, calculated at an equilibrium temperature of $1100^{\circ} \mathrm{C}$ with an assumed acceptor concentration of $5 \times 10^{-3}$ mol.\% (typical of nominally undoped $\mathrm{TiO}_{2}$ ) which is then quenched to room temperature. The defect concentrations from this cal- 
Table 1: Defect relations controlling the population of point defects in the annealed $\mathrm{TiO}_{2}$ crystals. The subscript $q$ refers to the quenched state [23, 24, 25, 26].

\begin{tabular}{|c|c|c|}
\hline $\begin{array}{c}\mathrm{H}\left(\mathrm{kJ} \mathrm{mol}^{-1}\right) \\
\mathrm{S}\left(\mathrm{J} \mathrm{mol}^{-1} \mathrm{~K}^{-1}\right)\end{array}$ & Annealing Equations & Quench Relations \\
\hline $\begin{array}{l}\mathrm{H}_{1}=493.1 \\
\mathrm{~S}_{1}=106.5\end{array}$ & {$\left[\mathrm{~V}_{\mathrm{O}}^{\bullet}\right]=\mathrm{K}_{1} n^{-2} p \mathrm{O}_{2}^{-1 / 2}$} & {$\left[\mathrm{~V}_{\mathrm{O}}^{\bullet}\right]_{q}=\left[\mathrm{V}_{\mathrm{O}}^{\bullet \bullet}\right]$} \\
\hline $\begin{aligned} \mathrm{H}_{2} & =879.2 \\
\mathrm{~S}_{2} & =190.8\end{aligned}$ & {$\left[\mathrm{Ti}_{\mathrm{i}}^{\bullet \bullet \bullet}\right]=\mathrm{K}_{2} n^{-3} p \mathrm{O}_{2}^{-1}$} & $\frac{\left[\mathrm{Ti}^{\dot{0 \bullet}]_{q}}\right.}{\left[\mathrm{Ti}_{\mathrm{i}}^{0 \bullet \bullet}\right]_{q}}=\frac{\mathrm{K}_{2 q} n_{q}}{\mathrm{~K}_{3 q}}$ \\
\hline $\begin{aligned} \mathrm{H}_{3} & =1025.8 \\
\mathrm{~S}_{3} & =238.3\end{aligned}$ & {$\left[\mathrm{Ti}_{\mathrm{i}}^{0 \cdots \cdots}\right]=\mathrm{K}_{3} n^{-4} p \mathrm{O}_{2}^{-1}$} & {$\left[\mathrm{Ti}_{\mathrm{i}}^{\bullet \bullet \bullet}\right]_{q}+\left[\mathrm{Ti}_{\mathrm{i}}^{\bullet \bullet \bullet \bullet}\right]_{q}=\left[\mathrm{Ti}_{\mathrm{i}}^{\bullet \bullet \bullet}\right]+\left[\mathrm{Ti}_{\mathrm{i}}^{\mathbf{\bullet} \bullet \bullet}\right]$} \\
\hline $\begin{array}{l}\mathrm{H}_{4}=354.5 \\
\mathrm{~S}_{4}=-202.1\end{array}$ & {$\left[\mathrm{~V}_{\mathrm{Ti}}^{\prime \prime \prime \prime}\right]=\mathrm{K}_{4} h^{-4} p \mathrm{O}_{2}$} & {$\left[\mathrm{~V}_{\mathrm{Ti}}^{\prime \prime \prime \prime}\right]_{q}=\left[\mathrm{V}_{\mathrm{Ti}^{\prime \prime \prime}}^{\prime \prime \prime}\right]$} \\
\hline $\begin{aligned} \mathrm{H}_{i} & =222.1 \\
\mathrm{~S}_{i} & =-44.6\end{aligned}$ & $h=\mathrm{K}_{i} n^{-1}$ & $h_{q}=\mathrm{K}_{i q} n_{q}^{-1}$ \\
\hline \multicolumn{3}{|c|}{$\mathrm{K}=\exp \left[(T S-H) / k_{B} T\right]$} \\
\hline
\end{tabular}

culation are subsequently used in the model for the phonon thermal conductivity to predict the change in phonon scattering by point defects in $\mathrm{TiO}_{2}$.

In reduced $\mathrm{TiO}_{2}\left(\mathrm{TiO}_{x}\right)$, when the level of substoichiometry is in the range of 1.999 $<x<1.9999$, the point defects tend to order into crystallographic shear planes termed "Wadsley" defects. Extremely reduced $\mathrm{TiO}_{x}, 1.67<x<1.999$, can have sufficient driving force to order the shear planes into Magnéli phases [27]. The homologous Magnéli phases have the general formula, $\mathrm{Ti}_{n} \mathrm{O}_{2 n-1}(3 \leq n \leq 38)$, and known shear planes are: $\{132\},\{121\}$ and $\{011\}[28,29]$.

According to the defect equilibrium calculations featured graphically in Fig. 1, the level of substoichiometry $\left(\mathrm{O} / \mathrm{Ti}\right.$ ratio) for heavily reduced samples at a $p \mathrm{O}_{2}$ of $4.2 \times 10^{-16} \mathrm{~atm}$ and $8.4 \times 10^{-18} \mathrm{~atm}$ are 1.99 and 1.98 , respectively. These values, 
however, are only estimates considering the uncertainty on temperature, $p \mathrm{O}_{2}$, and finite quench rates. Additionally, these calculations assume dilute solutions of defects and do not take into account interaction or ordering into extended defects or secondary phases. None the less, we anticipate that the samples annealed at a $\mathrm{pO}_{2}$ of $4.2 \times 10^{-16}$ atm are approaching the regime where shear planes will form and the samples annealed at a $\mathrm{pO}_{2}$ of $8.4 \times 10^{-18}$ atm are in the range of non-stoichiometry where Magnéli phases are expected to form.

Transmission electron microscopy (TEM) offers the ability to visualize the structure of the sample as well as analyze the diffraction pattern to gain information about defect phase formation. The atomic scale layering of Magnéli phases makes TEM an ideal means of investigating the structure of these materials, as seen by the wealth of such studies revolving around TEM in the literature $[30,29,31,32,33,34,8]$. In addition to direct visualization, TEM offers selected area diffraction patterns (SADP) to verify the crystallographic orientation and type of Magnéli phases as well. Evidence of these phases is seen in the form of crystallographically oriented features (streaking and weak diffraction peaks) in addition to the primary peaks in an SADP image $[29,31,32,33,34]$. To this extent, we verify the existence of these phases using TEM and SADP, seen in Sect. 4. To characterize the microstructure, site-selective TEM samples are prepared by focused ion beam (FIB) lift-out from specimens, reduced at $4.2 \times 10^{-16}$ atm and $8.4 \times 10^{-18}$ atm. The TEM samples are subsequently subjected to perform electron microscopy on JOEL 2000FX and 2010F transmission electron microscope operated at $200 \mathrm{kV}$.

Thermal conductivity measurements were conducted using time domain thermoreflectance (TDTR). TDTR is an optical pump-probe measurement technique that is ideal for analysis of thermal transport in thin films and semi-infinite planar samples $[35,36,37]$. The technique is based on analysis of changes in thermoreflectance of a thin metal film thermally excited and measured by an ultra-fast pulsed laser. An 80 $\mathrm{MHz}$ train of sub-picosecond pulses is split into a pump and probe path. The pump is amplitude modulated in the $0.5-8.8 \mathrm{MHz}$ range and frequency doubled to a wavelength of $400 \mathrm{~nm}$. The probe is time delayed using a mechanical delay stage and a lock-in amplifier is used to detect the frequency dependent change in thermoreflectance, mapping 
a thermal decay between pump pulses. This thermal decay measurement is fit to a multilayer heat transfer model in oder to determine the thermal properties of interest. In this study, the metal film used as a thermal transducer is $\mathrm{Al}$ deposited with electron-beam evaporation onto the crystal of interest. We use literature values of the volumetric heat capacities [38] and verify the thermal conductivity of the $\mathrm{Al}$ with a four point probe measurement. The main source of error in our analysis arrises from variation in the thickness of the $\mathrm{Al}$, which we use picosecond acoustics [39] and profilometry to verify at $64 \pm 3 \mathrm{~nm}$.

The penetration depth of our thermal measurement is dependent on the frequency of the modulated pump heating event. The relationship between the 1/e decay in the thermal gradient, the thermal properties of the material being studied, and the modulation frequencies is

$$
d_{\text {therm }}=\sqrt{\frac{\kappa}{\pi f C}}
$$

where $d_{\text {therm }}$ is the thermal penetration depth, $\kappa$ is the thermal conductivity, $C$ is the volumetric heat capacity, and $f$ is the modulation frequency of the pump pulses. In order to verify that the thermal measurements that we conduct are not dictated by the near-surface nature of our measurement, we probe various thermal penetration depths to ensure that there is no depth dependence in the measured thermal conductivity of the crystals. The modulation frequencies that we use range from $8.8 \mathrm{MHz}$ to 500 $\mathrm{kHz}$, corresponding to thermal penetration depths in $\mathrm{TiO}_{2}$ ranging roughly from 300 $\mathrm{nm}$ to $1.3 \mu \mathrm{m}$. The spatial dimension of our measurement in the lateral dimension is dictated by the laser spot size with diameters of $27 \mu \mathrm{m}$ and $17 \mu \mathrm{m}$ for the pump and probe respectively. With these relatively large spot sizes, a wide range of thermal penetration depth, and measurement of multiple regions of the crystals, we ensure that any variations in individual measurements are statistical in nature, and not owing to underlying changes in thermal physics [35, 36, 37, 40, 41].

We obtain measurements for the thermal conductivity of the crystals in both the [100] and [001] crystallographic directions by using two different single crystal samples with the desired surface normal, both subjected to the same processing. Each 
sample was coated with an $\mathrm{Al}$ transducer on the $10 \mathrm{~mm} \times 10 \mathrm{~mm}$ polished face and the

TDTR measurement was conducted to determine the cross-plane thermal conductivity.

\section{Thermal conductivity model}

In order to further understand the trends in the thermal conductivity of defected functional oxides, we develop an analytical model for the phonon thermal conductivity of reduced $\mathrm{TiO}_{2}$. Our model consists of an integration over phonon wave vectors for each branch in the phonon dispersion as follows

$$
\kappa=\sum_{i} \int_{k} C_{i} v_{i}^{2} \tau_{i} d k
$$

where the thermal conductivity, $\kappa$, is the product of the heat capacity, $C$, the phonon group velocity, $v$ and the characteristic scattering time, $\tau$. All of these components are phonon frequency (or wave vector, $k$ ) dependent with relationships dictated by the phonon dispersion relationship for each branch, $i$ (with 15 total branches; 3 acoustic and 12 optical).

Since rutile has a tetragonal unit cell, we assume a cylindrical Brillouin zone and define the phonon frequency, $\omega$, as

$$
\begin{aligned}
\omega\left(k_{x}, k_{y}, k_{z}\right) & =\sqrt{\omega\left(k_{x}\right)^{2}+\omega\left(k_{y}\right)^{2}+\omega\left(k_{z}\right)^{2}} \\
& =\sqrt{2 \omega\left(k_{x}\right)^{2}+\omega\left(k_{z}\right)^{2}}
\end{aligned}
$$

where $k_{x}, k_{y}$, and $k_{z}$ are the phonon wave vectors in the $\Gamma \rightarrow X, \Gamma \rightarrow Y$, and $\Gamma \rightarrow Z$ direction, respectively. In this model, we use a polynomial fit to a dispersion relationship that has been determined by lattice dynamics [42].

The general formulation for the heat capacity of a crystal can be written as

$$
C=\sum_{i} \frac{1}{8 \pi^{3}} \int_{k_{x}} \int_{k_{y}} \int_{k_{z}} \hbar \omega_{i} \frac{d f}{d T} d k_{x} d k_{y} d k_{z}
$$


where $\hbar \omega$ is the phonon energy dictated by the product of Planck's constant and the phonon frequency, and $\frac{d F}{d T}$ is the change in the Bose-Einstein distribution with temperature. In an isotropic solid, a spherical Brillouin zone is often assumed, leading to

$$
\begin{aligned}
C & =\sum_{i} \frac{1}{8 \pi^{3}} \int_{0}^{2 \pi} \int_{0}^{\pi} \int_{k} \hbar \omega_{i} \frac{d f}{d T} k^{2} \sin (\theta) d k d \phi d \theta \\
& =\sum_{i} \frac{1}{8 \pi^{2}} \int_{k} \hbar \omega \frac{d F}{d T} k^{2} d k
\end{aligned}
$$

where the only spatial term that dictates the heat capacity is the radius of the Brillouin Zone, or the phonon wave vector.

Using our aforementioned crylindrical approximation for the Brillouin Zone, the heat capacity is defined by

$$
C=\sum_{i} \frac{1}{8 \pi^{3}} \int_{0}^{2 \pi} \int_{k_{r}} \int_{-k_{z}}^{k_{z}} \hbar \omega_{i} \frac{d f}{d T} k_{r} d k_{z} d k_{r} d \theta
$$

where $k_{z}$ is the wave vector associated with the anisotropic axis, [001] in the case of $\mathrm{TiO}_{2}$, and $k_{r}$ is the wave vector in isotropic plane, formulated as $k_{r}=\sqrt{k_{x}^{2}+k_{y}^{2}}$ where $k_{x}$ and $k_{y}$ are the wave vectors in the [100] and [010] directions in $\mathrm{TiO}_{2}$. By symmetry, this simplifies to

$$
C=\sum_{i} \frac{1}{2 \pi^{2}} \int_{k_{r}} \int_{k_{z}} \hbar \omega_{i} \frac{d f}{d T} k_{r} d k_{z} d k_{r}
$$

The other components of the thermal conductivity are the phonon group velocity, which is determined by the first derivative of the phonon frequency with respect to wave number, and the phonon scattering time. The total phonon scattering time is modeled using Matthissein's rule,

$$
\frac{1}{\tau}=\frac{1}{\tau_{P h}}+\frac{1}{\tau_{\text {Imp }}}+\frac{1}{\tau_{\text {Bound }}}+\frac{1}{\tau_{\text {Instl }}}+\frac{1}{\tau_{\text {Vac }}}
$$

where the total scattering time, $\tau$ is dictated by the phonon-phonon scattering time, $\tau_{P h}$,

the scattering of phonons with impurities, $\tau_{\text {Imp }}$, the phonon-boundary scattering time, 
$\tau_{\text {Bound }}$, and the scattering of phonons with intrinsic point defects such as interstitials, $\tau_{\text {Instl }}$, and vacancies, $\tau_{V a c}$.

In our experiment, we assume that the first three scattering mechanisms are constant within our sample set and have a functional form as follows

$$
\frac{1}{\tau_{\text {Baseline }}}=\mathscr{A} T \omega^{2} \exp \left(\frac{-\mathscr{B}}{T}\right)+\mathscr{C} \omega^{4}+\frac{v}{d}
$$

185

where $T$ is the sample temperature, $d$ is the sample thickness, and $\mathscr{A}, \mathscr{B}$, and $\mathscr{C}$ are fitting parameters. We determine $\mathscr{A}$ and $\mathscr{B}$ using a least squares fit of literature values of measured thermal conductivity over a wide temperature range [43] and modify $\mathscr{C}$ to fit the measured values of the control sample in our specific sample set.

The formulation for scattering from known concentrations of point defects takes the form of Rayleigh scattering $\left(\propto \omega^{4}\right)$ and is determined by mass differences, size differences, and bond strength differences on the atomic level $[15,14,19]$.

$$
\frac{1}{\tau_{D e f}}=\frac{a^{3}}{4 \pi v^{3}} x_{D e f} \omega^{4}\left[\left(\frac{\Delta M}{M}\right)^{2}+2\left[\left(\frac{\Delta G}{G}\right)-6.4 \gamma\left(\frac{\Delta \delta}{\delta}\right)\right]^{2}\right]
$$

where $a^{3}$ is the unit cell volume, $x_{D e f}$ is the atomic concentration of the defect of interest, $\gamma$ is the Grüneisen parameter, and $\Delta M / M, \Delta G / G$, and $\Delta \delta / \delta$ are the changes in mass, shear strength, and atomic radius, respectively, of the defect compared to the host. In the case of a substitutional or interstitial defect, this parameter takes the form

$$
\frac{\Delta M}{M}=\frac{M_{\text {Def }}-\bar{M}_{\text {Host }}}{\bar{M}_{\text {Host }}}
$$

where $M_{D e f}$ is the mass of the defect and $\bar{M}_{\text {Host }}$ is the average atomic mass of the host. In the case of a vacancy, this takes the form [17]

$$
\frac{\Delta M_{V a c}}{M}=-\frac{M_{V a c}}{\bar{M}_{H o s t}}-2
$$

where $M_{V a c}$ is the mass of the vacant atom. In the case of the interstitial defects, the change in radius is determined by the difference between the radius of the interstitial site in rutile $\mathrm{TiO}_{2}$ [44] and the radius of the anion [45]. 


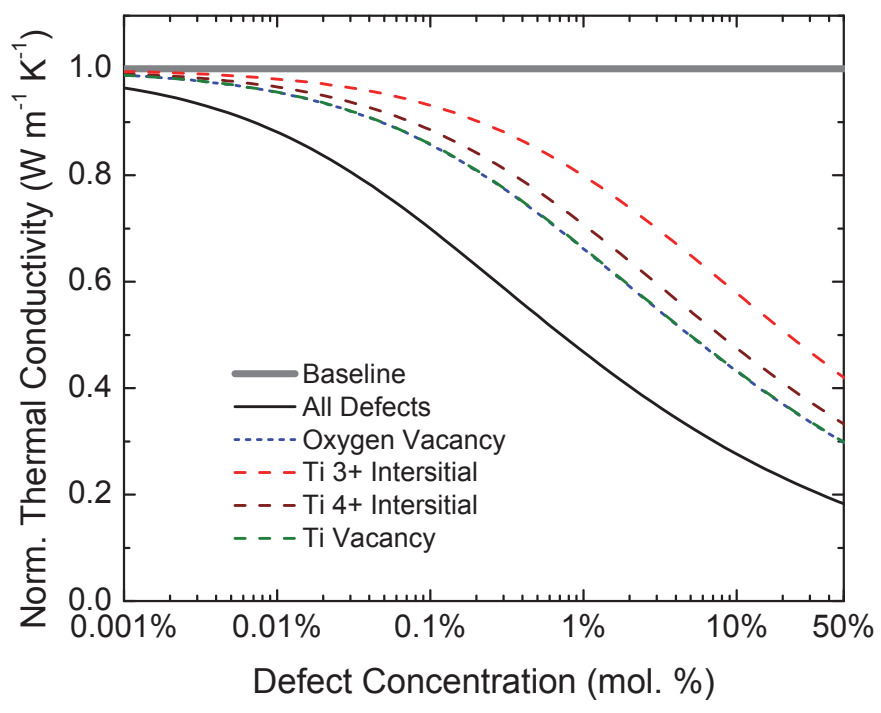

Figure 2: Normalized thermal conductivity with varying concentrations of titanium interstitials $\left(\mathrm{Ti}^{3+}\right.$ and $\mathrm{Ti}^{4+}$ ), titanium vacancies, and oxygen vacancies (as well as a simple linear summation of all four), determined by the analytical model for phonon thermal conductivity described in Sect. 3 .

The physical parameters used for each type of defect accounted for in this model were taken from literature $[45,46,47]$. The impact of intrinsic defects on the thermal conductivity of rutile $\mathrm{TiO}_{2}$ can be seen in Fig. 2 for a wide range of concentrations of titanium interstitials $\left(\mathrm{Ti}^{3+}\right.$ and $\mathrm{Ti}^{4+}$ ), titanium vacancies, and oxygen vacancies (as well as a simple linear summation of all four). Comparing this model to the results from our measurements, we can use inputs determined by the equilibrium concentration at the anneal temperature, seen in Fig. 1, and determine the expected impact of intrinsic defects in highly reduced $\mathrm{TiO}_{2}$ on thermal transport. From this it is clear that in the operating range of $0.5-1$ mol. $\%$ concentration of point defects, we expect a measurable (i.e. greater than $10 \%$ ) change in thermal conductivity.

\section{Results \& Discussion}

The measurements of the thermal conductivity of reduced $\mathrm{TiO}_{2}$ single crystals can be seen in Fig. 3 as solid points. In accordance with literature findings [43, 48], we observe significant anisotropy in the thermal conductivity of rutile along the principal 


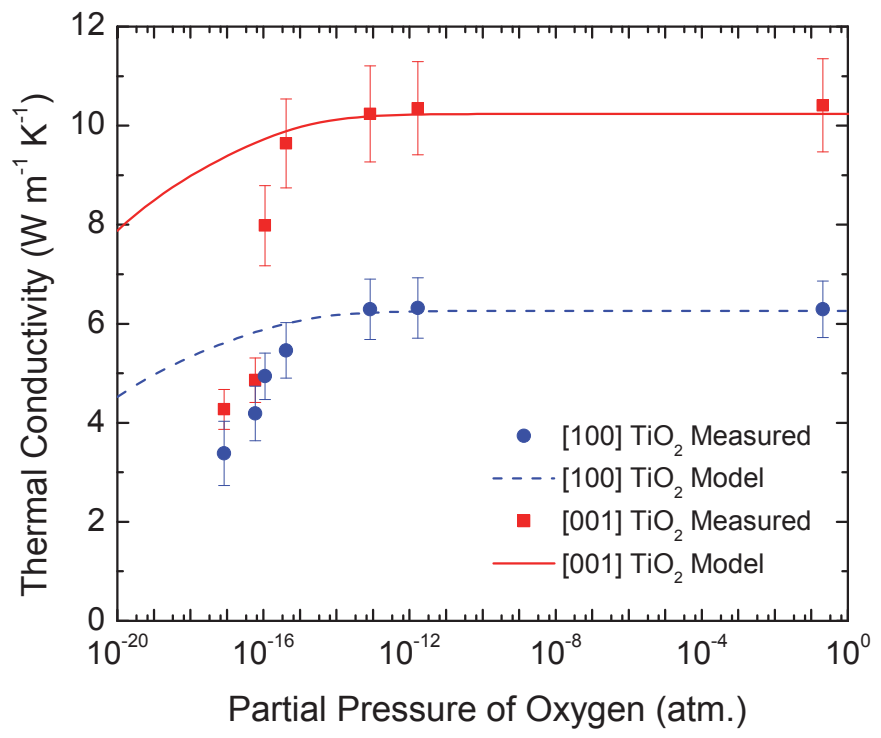

Figure 3: Thermal conductivity of [100]-oriented and [001]-oriented rutile $\mathrm{TiO}_{2}$ with various defect concentrations dictated by $p \mathrm{O}_{2}$ during anneals. The dotted lines indicate the modeled impact of various defects on the thermal conductivity using defect concentrations found in Fig. 1.

directions $\left(6.3 \pm 0.6 \mathrm{~W} \mathrm{~m}^{-1} \mathrm{~K}^{-1}\right.$ in the [100] direction and $10.4 \pm 0.9 \mathrm{~W} \mathrm{~m}^{-1} \mathrm{~K}^{-1}$ in the [001] direction). The thermal conductivity of the rutile crystals is relatively unaffected by any change in defect concentration until processed at a $\mathrm{O}_{2}$ of $10^{-14}$ atm. Below this processing threshold, the defect concentration induced by low $p \mathrm{O}_{2}$ during annealing is significant enough to decrease the thermal conductivity in both orientations. This threshold corresponds to a total defect concentration of $\sim 0.5 \mathrm{~mol} \%$, taking into account the major intrinsic defect types discussed previously. Furthermore, the anisotropy from the rutile crystal structure is diminished at the samples with the lowest $\mathrm{pO}_{2}$ levels with only a $15-20 \%$ difference between the thermal conductivity measured in the [100] and [001] directions.

The model for the thermal conductivity of rutile dictated by $p \mathrm{O}_{2}$ equilibration levels shown by the curves in Fig. 3 accurately predicts the threshold of impact of defect concentration on the thermal conductivity (no significant change for $p \mathrm{O}_{2}$ above $\left.10^{-14} \mathrm{~atm}\right)$. However, the data departs from the expected model at $p \mathrm{O}_{2}$ levels below 

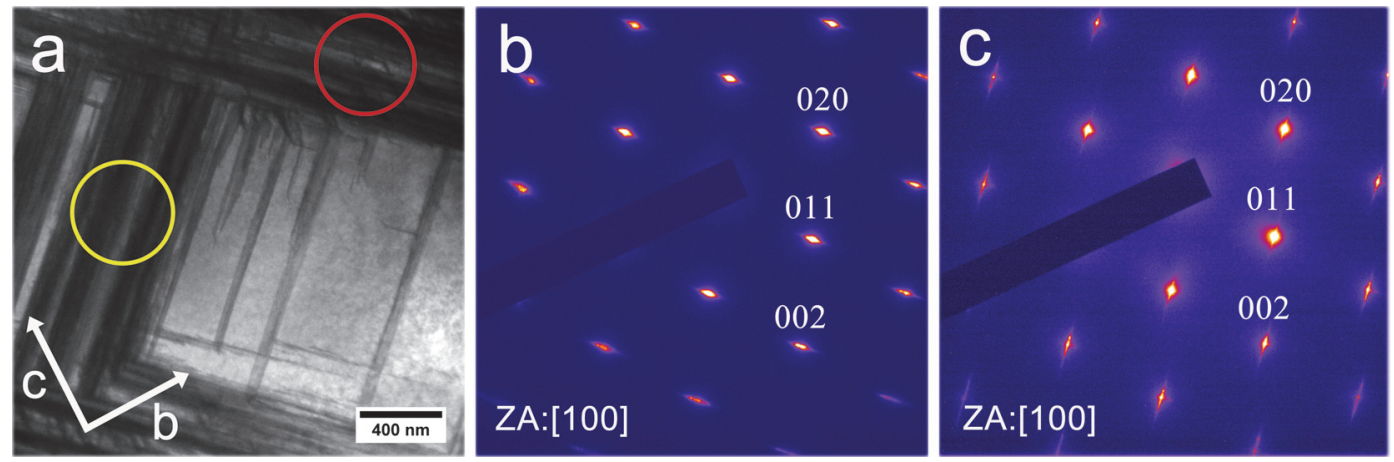

Figure 4: (a) $<100>$ zone axis bright field transmission electron microscopy (BF-TEM) image of the $\mathrm{TiO}_{1.99}$ specimen showing two Wadsley defect regions, orientation of the rutile crystal are indicated by the arrows showing the b, or [010], direction and c, or [001] direction. (b and c) Selected area diffraction patterns (SADPs) at $<100>$ zone axis from two regions, oriented in different directions, indicating elongation of diffracted spots in the $<064>$ direction.

$4.2 \times 10^{-16} \mathrm{~atm}$. With a total concentration of defects exceeding $1 \mathrm{~mol} . \%$, the system is sub-stoichiometric to a degree that we expect the formation of planar shear defects. If extended defects are indeed forming at these extreme $p \mathrm{O}_{2}$ levels, then our data suggest that these layered phases reduce the thermal conductivity well beyond the expected impact from non-interacting point defects. Furthermore, at the lowest $p \mathrm{O}_{2}$ levels that we could achieve (as low as $8.4 \times 10^{-18}$ atm), the anisotropy in the thermal conductivity is no longer present in our measured data. This suggests that the symmetry of the crystal has changed. The existence of Wadsley defects and Magnéli phases at extreme annealing conditions is verified using TEM seen in Fig. 4 and Fig. 5.

Figure 4 shows a [100] zone axis bright field transmission electron microscopy (BF-TEM) image of the $\mathrm{TiO}_{2}$ specimen, annealed at $4.2 \times 10^{-16} \mathrm{~atm}$, showing Wadsley defect regions oriented in two different directions with respect to the surface normal [001], probed during TDTR. Figures $4 \mathrm{~b}$ and $4 \mathrm{c}$ are selected area diffraction patterns (SADP) along [100] zone axis from the regions are specified in the Fig. 4a with yellow circle and red circle, respectively. In both diffraction patterns, the diffracted spots are elongated in the $\mathrm{g}_{064}$ directions in reciprocal space. Ordered superlattice reflections were not observed for other zone-axis orientations, including [310] and [210] indicating that the stoichiometry corresponds to $\mathrm{x}>1.999$. The TEM microscopy results 

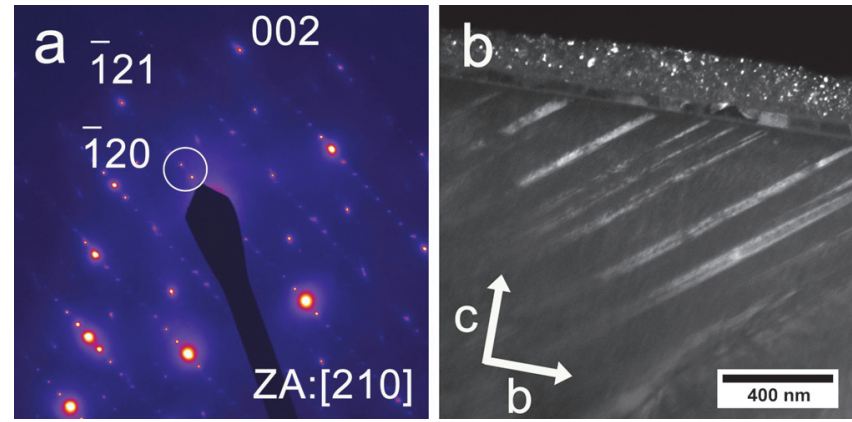

Figure 5: (a) Diffraction pattern of $\mathrm{TiO}_{1.98}$ specimen at $<210>$ zone axis showing superlattice reflections along $\mathrm{g}\{121\}$, (b) Dark-field micrograph of $\mathrm{TiO}_{1.98}$ specimen formed by superlattice reflections, showing oriented planar defects, orientation of the rutile crystal are indicated by the arrows showing the b, or [010], direction and c, or [001] direction.

are consistent with $\{132\}$-type shear planes that are inclined respect to the zone axis, however, these planar defects are not periodically ordered to form Magnéli phases.

Figure 5a, a diffraction pattern from the extremely reduced sample (annealed at $\left.8.4 \times 10^{-18} \mathrm{~atm}\right)$, taken at [210] zone axis, exhibits superlattice reflections associated with point defect ordering into the Magnéli phases. The arrangement of superlattice reflection along $<121>$ directions in reciprocal space, supports Magnéli phase formation with $\{121\}$ type of shear planes (Fig. 5b). In this region of the sample, the periodicity of the superlattice reflections equals $8 \mathrm{~d}_{121}$, which corresponds to the Magnéli phase $\mathrm{Ti}_{8} \mathrm{O}_{15}$ (or $\mathrm{TiO}_{1.875}$ ). A dark field image is also obtained from the superlattice reflections (shown in Fig. 5a), highlighting the regions of the sample containing Magnéli phases with that particular crystallographic orientation (bright regions in Fig. 5b). As there are eight variants of the $\{121\}$ shear planes with respect to the rutile crystal axes, multiple domain variants are expected to form during reduction of the crystal. This sample is no longer single crystalline.

Our investigations indicate that the formation of these secondary phases occurs inhomogeneously, a notion that is verified by literature findings which have shown it to be kinetically difficult to achieve a single phase Magnéli structure when reducing from a single crystal [27]. Thus the measured thermal transport is not intrinsic to the $\mathrm{Ti}_{8} \mathrm{O}_{15}$ Magnéli phase since domain (and possibly phase) boundaries are present within 
the interrogated volume of sample. These multiple domains further explain the loss in measured anisotropy between crystals of orthogonal orientations. The highly ordered crystallites of Magnéli phases that are forming will not be preferentially aligned to the orientation of our thermal measurement, and so we will no longer be able to resolve the previously clear anisotropy. This evidence of the presence of the extended defects and Magnéli phases in highly reduced $\mathrm{TiO}_{2}$ explains the departure from the expected trend in the thermal conductivity of reduced rutile is due to increased phonon scattering from interaction with the nanoscale boundaries associated with Wadsley defects and Magnéli phases.

\section{Conclusions}

We have shown clear evidence of the interaction of intrinsic point defects with phonons in single crystal, rutile $\mathrm{TiO}_{2}$. Control over the intrinsic point defect profile was accomplished using high temperature anneals and regulated partial pressures of oxygen. At low partial pressures of oxygen, the total intrinsic defect concentration is driven well beyond 1 mol.\% resulting in a significant reduction in the thermal conductivity and loss of the measured anisotropic thermal properties.

As the sub-stoichiometry becomes severe, the rutile $\mathrm{TiO}_{2}$ undergoes an ordering of point defects into crystallographically oriented shear planes, and a phase transition into layered Magnéli phases. This phase transition results in a reduction in the thermal conductivity that is well beyond that expected from a homogeneous, non-interacting profile of point defects. These findings have significance particularly to the application of dielectric breakdown and resistive switching, in which charged point defects drift and concentrate near electrodes during prolonged application of high electric fields in dielectric crystals [8].

\section{Acknowledgements}

BFD and PEH appreciate support from AFOSR grant number FA9550-14-1-0067. ECD, DL and AM acknowledge support from the National Science Foundation under 
grant number DMR-1132058 and the NCSU Analytical Instrumentation Facility for access to the electron microscopy facilities.

[1] S. Lombardo, J. H. Stathis, B. P. Linder, K. L. Pey, F. Palumbo, C. H. Tung, Dielectric breakdown mechanisms in gate oxides, Journal of Applied Physics 98 (12) (2005) 121301. doi:http://dx.doi.org/10.1063/1. 2147714 .

URL http://scitation.aip.org/content/aip/journal/jap/ $98 / 12 / 10.1063 / 1.2147714$

[2] S. Blonkowski, Filamentary model of dielectric breakdown, Journal of Applied Physics 107 (8). doi:http://dx.doi.org/10.1063/1.3386517.

URL http://scitation.aip.org/content/aip/journal/jap/ $107 / 8 / 10.1063 / 1.3386517$

[3] S. Whitehead, W. Nethercot, The breakdown of dielectrics under high voltage, with particular reference to thermal instability, Proceedings of the Physical Society 47 (5) (1935) 974.

URL http://stacks.iop.org/0959-5309/47/i=5/a=322

[4] J. O'Dwyer, Theory of dielectric breakdown in solids, Journal of The Electrochemical Society 116 (2) (1969) 239-242.

[5] R. Waser, T. Baiatu, K.-H. Härdtl, dc electrical degradation of perovskite-type titanates: I, ceramics, Journal of the American Ceramic Society 73 (6) (1990) 1645-1653. doi:10.1111/j.1151-2916.1990.tb09809.x.

URL http://dx.doi.org/10.1111/j.1151-2916.1990. tbo9809.x

[6] R. Waser, T. Baiatu, K.-H. Härdtl, dc electrical degradation of perovskite-type titanates: II, single crystals, Journal of the American Ceramic Society 73 (6) (1990) 1654-1662. doi:10.1111/j.1151-2916.1990.tb09810.x. URL http://dx.doi.org/10.1111/j.1151-2916.1990. tbo9810.x 
[7] T. Baiatu, R. Waser, K.-H. Härdtl, dc electrical degradation of perovskite-type titanates: III, a model of the mechanism, Journal of the American Ceramic Society 73 (6) (1990) 1663-1673. doi:10.1111/j.1151-2916.1990. tb09811.x.

[12] J. McPherson, J.-Y. Kim, A. Shanware, H. Mogul, Thermochemical 
[18] H. Siebeneck, W. P. Minnear, R. C. Bradt, D. P. H. Hasselman, Thermal diffusivity of nonstoichiometric titanium dioxide, Journal of the American Ceramic Society 59(1-2)(1976)84-84. doi:10.1111/j.1151-2916.1976.tb09401.x. URL http://dx.doi.org/10.1111/j.1151-2916.1976. tbo9401.x

als, Applied Physics Letters 82 (13) (2003) 2121-2123. doi:http: //dx.doi.org/10.1063/1.1565180.

URL http://scitation.aip.org/content/aip/journal/apl/ $82 / 13 / 10.1063 / 1.1565180$

[13] R. J. Kamaladasa, A. A. Sharma, Y.-T. Lai, W. Chen, P. A. Salvador, J. A. Bain, M. Skowronski, Y. N. Picard, In situ tem imaging of defect dynamics under electrical bias in resistive switching rutile-tio2, Microscopy and Microanalysis 21 (2015) 140-153. doi:10.1017/S1431927614013555.

URL http://journals.cambridge.org/article_ S1431927614013555

[14] P. G. Klemens, The scattering of low-frequency lattice waves by static imperfections, Proceedings of the Physical Society. Section A 68 (12) (1955) 1113.

URL http://stacks.iop.org/0370-1298/68/i=12/a=303

[15] B. Abeles, Lattice thermal conductivity of disordered semiconductor alloys at high temperatures, Phys. Rev. 131 (1963) 1906-1911. doi:10.1103/ PhysRev.131.1906.

URL http://link.aps.org/doi/10.1103/PhysRev.131.1906

[16] P. G. Klemens, Theory of heat conduction in nonstoichiometric oxides and carbides, High Temperatures - High Pressures 17 (1) (1985) 41-45.

[17] C. Ratsifaritana, P. Klemens, Scattering of phonons by vacancies, International Journal of Thermophysics 8 (6) (1987) 737-750. doi:10.1007/ BF 00500791 .

URL http://dx.doi.org/10.1007/BF00500791 
[19] P. G. Klemens, Phonon scattering by oxygen vacancies in ceramics, Physica B: Condensed Matter 263-264 (0) (1999) 102 - 104. doi:http://dx.doi.org/10.1016/s0921-4526(98)01202-2. URL http://www.sciencedirect.com/science/article/pii/ S0921452698012022

[20] C. M. Brooks, R. B. Wilson, A. Schafer, J. A. Mundy, M. E. Holtz, D. A. Muller, J. Schubert, D. G. Cahill, D. G. Schlom, Tuning thermal conductivity in homoepitaxial $\mathrm{SrTiO}_{3}$ films via defects, Applied Physics Letters 107 (5) (2015) -. doi:http://dx.doi.org/10.1063/1.4927200.

URL http://scitation.aip.org/content/aip/journal/apl/ $107 / 5 / 10.1063 / 1.4927200$

[21] S. Harada, K. Tanaka, H. Inui, Thermoelectric properties and crystallographic shear structures in titanium oxides of the magneli phases, Journal of Applied Physics 108 (8) (2010) -. doi:http://dx.doi.org/10.1063/1. 3498801 .

URL http://scitation.aip.org/content/aip/journal/jap/ $108 / 8 / 10.1063 / 1.3498801$

[22] R. Swalin, Thermodynamics of solids, Wiley series on the science and technology of materials, Wiley, 1962.

URL https://books.google.com/books?id=8DxRAAAAMAAJ

[23] M. Nowotny, T. Bak, J. Nowotny, Electrical properties and defect chemistry of $\mathrm{TiO}_{2}$ single crystal. III. equilibration kinetics and chemical diffusion, The Journal of Physical Chemistry B 110 (33) (2006) 16292-16301.

[24] J. Nowotny, T. Bak, M. K. Nowotny, L. R. Sheppard, Defect chemistry and electrical properties of titanium dioxide. 1. defect diagrams, The Journal of Physical Chemistry C 112 (2) (2008) 590-601. arXiv:http://dx.doi.org/10 . 1021/jp074565u, doi:10.1021/jp074565u. URL http://dx.doi.org/10.1021/jp074565u 
[25] J. Nowotny, M. A. Alim, T. Bak, M. A. Idris, M. Ionescu, K. Prince, M. Z. Sahdan, K. Sopian, M. A. Mat Teridi, W. Sigmund, Defect chemistry and defect engineering of $\mathrm{TiO}_{2}$-based semiconductors for solar energy conversion, Chem. Soc. Rev. 44 (2015) 8424-8442. doi:10.1039/C4CS00469H.

URL http://dx.doi.org/10.1039/C4CS00469H

[26] D. M. Smyth, The defect chemistry of metal oxides, The Defect Chemistry of Metal Oxides, by DM Smyth, pp. 304. Foreword by DM Smyth. Oxford University Press, Jun 2000. ISBN-10: 0195110145. ISBN-13: 97801951101421.

[27] K. Szot, M. Rogala, W. Speier, Z. Klusek, A. Besmehn, R. Waser, $\mathrm{TiO}_{2}$ - a prototypical memristive material, Nanotechnology 22 (25) (2011) 254001.

URL http://stacks.iop.org/0957-4484/22/i=25/a=254001

[28] L. Bursill, B. Hyde, Crystallographic shear in the higher titanium oxides: Structure, texture, mechanisms and thermodynamics, Progress in Solid State Chemistry 7 (1972) 177 - 253. doi:http://dx.doi.org/10.1016/ $0079-6786(72) 90008-8$.

URL http://www.sciencedirect.com/science/article/pii/ 0079678672900088

[29] L. A. Bursill, B. G. Hyde, O. Terasaki, D. Watanabe, On a new family of titanium oxides and the nature of slightly-reduced rutile, Philosophical Magazine 20 (164) (1969) 347-359. arXiv:http://dx.doi.org/10.1080/ 14786436908228706 , doi:10.1080/14786436908228706.

URL http://dx.doi.org/10.1080/14786436908228706

[30] L. A. Bursill, B. G. Hyde, D. K. Philp, New crystallographic shear fami430 lies derived from the rutile structure, and the possibility of continuous ordered solid solution, Philosophical Magazine 23 (186) (1971) 1501-1513. arXiv:http://dx.doi.org/10.1080/14786437108217017, doi: $10.1080 / 14786437108217017$.

URL http://dx.doi.org/10.1080/14786437108217017 
[37] P. E. Hopkins, J. R. Serrano, L. M. Phinney, S. P. Kearney, T. W. Grasser, C. T. Harris, Criteria for cross-plane dominated thermal transport in multilayer thin 
film systems during modulated laser heating, Journal of Heat Transfer 132 (8)

[38] M. Chase, NIST-JANAF Thermochemical Tables, Vol. 1, National Institute of Standards and Technology, 1998.

[39] C. Thomsen, J. Strait, Z. Vardeny, H. J. Maris, J. Tauc, J. J. Hauser, Coherent (2010) 081302-081302.

URL http://dx.doi.org/10.1115/1.4000993 phonon generation and detection by picosecond light pulses, Phys. Rev. Lett. 53 (1984) 989-992. doi:10.1103/PhysRevLett.53.989.

URL http://link.aps.org/doi/10.1103/PhysRevLett.53.989

[40] Y. K. Koh, D. G. Cahill, Frequency dependence of the thermal conductivity of semiconductor alloys, Phys. Rev. B 76 (2007) 075207. doi:10.1103/ PhysRevB.76.075207.

URL http://link.aps.org/doi/10.1103/PhysRevB.76.075207

[41] R. B. Wilson, D. G. Cahill, Anisotropic failure of fourier theory in time-domain thermoreflectance experiments, Nature Communications 5 (2014) 5075 EP -. URL http://dx.doi.org/10.1038/ncomms 6075

[42] J. G. Traylor, H. G. Smith, R. M. Nicklow, M. K. Wilkinson, Lattice dynamics of rutile, Phys. Rev. B 3 (1971) 3457-3472. doi:10.1103/PhysRevB.3. 3457.

URL http://link.aps.org/doi/10.1103/PhysRevB.3.3457

[43] W. R. Thurber, A. J. H. Mante, Thermal conductivity and thermoelectric power of rutile $\left(\mathrm{TiO}_{2}\right)$, Phys. Rev. 139 (1965) A1655-A1665. doi:10.1103/ PhysRev.139.A1655. URL http://link.aps.org/doi/10.1103/PhysRev.139.A1655

[44] E. M. Golden, N. C. Giles, S. Yang, L. E. Halliburton, Interstitial silicon ions in rutile $\mathrm{TiO}_{2}$ crystals, Phys. Rev. B 91 (2015) 134110. doi:10.1103/ PhysRevB.91.134110.

URL http://link.aps.org/doi/10.1103/PhysRevB.91.134110 
[45] R. D. Shannon, Revised effective ionic radii and systematic studies of interatomic distances in halides and chalcogenides, Acta Crystallographica Section A 32 (5) (1976) 751-767. doi :10.1107/S0567739476001551. URL http://dx.doi.org/10.1107/S0567739476001551

[46] R. M. Hazen, L. W. Finger, Bulk moduli and high-pressure crystal structures of rutile-type compounds, Journal of Physics and Chemistry of Solids 42 (3) (1981) 143 - 151. doi:http://dx.doi.org/10.1016/0022-3697(81) 90074-3.

URL http://www.sciencedirect.com/science/article/pii/ 0022369781900743

[47] D. G. Isaak, J. D. Carnes, O. L. Anderson, H. Cynn, E. Hake, Elasticity of $\mathrm{TiO}_{2}$ rutile to $1800 \mathrm{~K}$, Physics and Chemistry of Minerals 26 (1) (1998) 31-43. doi : $10.1007 / \mathrm{s} 002690050158$. URL http://dx.doi.org/10.1007/s002690050158

[48] E. Shojaee, M. R. Mohammadizadeh, First-principles elastic and thermal properties of $\mathrm{TiO}_{2}$ : a phonon approach, Journal of Physics: Condensed Matter 22 (1) (2010) 015401.

URL http://stacks.iop.org/0953-8984/22/i=1/a=015401 

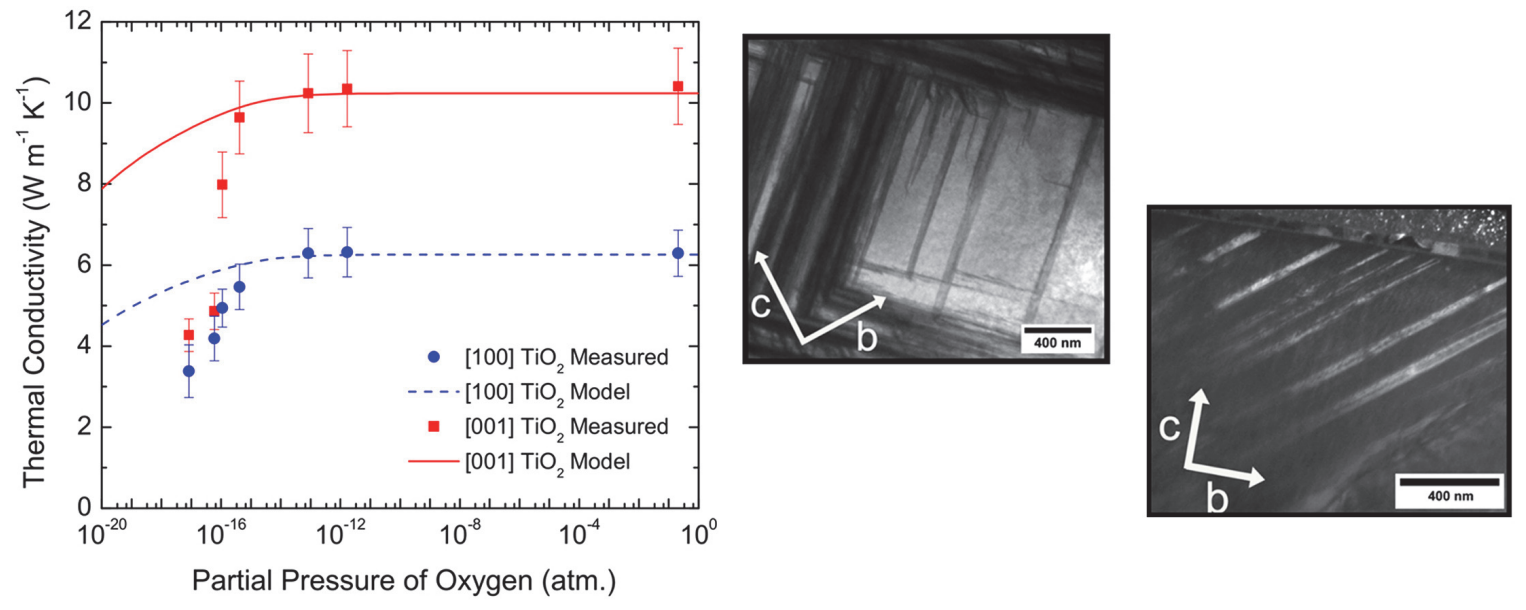